\title{
A blockchain-based smart contract trading mechanism for energy power supply and demand network
}

\author{
Hu, W. ${ }^{a}$, Hu, Y.W. ${ }^{a}{ }^{*}$, Yao, W.H. ${ }^{a}$, Lu, W.Q. ${ }^{a}$, Li, H.H. ${ }^{a}$, Lv, Z.W. ${ }^{a}$ \\ ${ }^{a}$ School of Economics and Management, Shanghai University of Electric Power, Shanghai, P.R. China
}

\begin{abstract}
A B S T R A C T
To overcome the high cost, high risk and poor efficiency of traditional centralized electric energy trading method, this paper proposes an efficient trading mechanism for energy power supply and demand network (EPSDN) based on blockchain smart contract, considering the opening of the sales side market in China. Specifically, the encourage-real-quotation (ERQ) rule was adopted to determine the clearing queue and price, thus smoothing the supply and demand interaction between the EPSDN node. Meanwhile, the blockchain smart contract was introduced into the transaction to form a sealed quotation function, which eliminates the centralization and high cost and solves the poor transparency and trust in traditional transaction. In addition, the transaction efficiency was improved through the construction of an efficient power trading system and a secure trading environment. A case study is given in the end of the paper. Case study shows that the blockchain-based smart contract trading system for the EPSDN can achieve desirable security and effectiveness, and effectively solve the problems of the traditional centralized trading method. The research findings lay solid theoretical and decision-making bases for small-scale transactions in the electric energy market.
\end{abstract}

(C) 2019 CPE, University of Maribor. All rights reserved.

\author{
ARTICLE INFO \\ Keywords: \\ Electric energy; \\ Energy power supply and demand \\ network (EPSDN); \\ Blockchain; \\ Smart contract: \\ Encourage-real-quotation (ERQ) \\ rule; \\ Power transaction \\ *Corresponding author: \\ 99030411@qq.com \\ (Hu, Y.W.) \\ Article history: \\ Received 18 April 2019 \\ Revised 7 September 2019 \\ Accepted 9 September 2019
}

\section{References}

[1] Liu, Z. (2015). Global energy internet, China Electric Power Press, Beijing, China.

[2] Huckle, S., Bhattacharya, R., White, M., Beloff, N. (2016). Internet of things, blockchain and shared economy applications, Procedia Computer Science, Vol. 98, 461-466, doi: 10.1016/j.procs.2016.09.074.

[3] Sikorski, J.J., Haughton, J., Kraft, M. (2017). Blockchain technology in the chemical industry: Machine-to-machine electricity market, Applied Energy, Vol. 195, 234-246, doi: 10.1016/j.apenergy.2017.03.039.

[4] Chiu, Y.-S.P., Chen, H.-Y., Chiu, T., Chiu, S.W. (2018). Incorporating flexible fabrication rate and random scrap into a FPR-based supply-chain system, Economic Computation and Economic Cybernetics Studies and Research, Vol. 52, No. 2, 157-174, doi: 10.24818/18423264/52.2.18.10.

[5] Sharma, P.K., Moon, S.Y., Park, J.H. (2017). Block-VN: A distributed blockchain based vehicular network architecture in smart city, Journal of Information Processing Systems, Vol. 13, No. 1, 184-195, doi: 10.3745/】IPS.03.0065.

[6] Lalami, I., Frein, Y., Gayon, J.P. (2017). Demand variability and value of information sharing in the supply chain. A case study in the automotive industry, Journal Européen des Systèmes Automatisés, Vol. 50, No. 1-2, 157-186, doi: 10.3166/JESA.50.157-186.

[7] Kshetri, N. (2017). Can blockchain strengthen the internet of things?, IT Professional, Vol. 19, No. 4, 68-72, doi: 10.1109/MITP.2017.3051335.

[8] Hussein, A.F., Arunkumar, N., Ramirez-Gonzalez, G., Abdulhay, E., Tavares, J.M.R.S., De Albuquerque, V.H.C. (2018). A medical records managing and securing blockchain based system supported by a genetic algorithm and discrete wavelet transform, Cognitive Systems Research, Vol. 52, 1-11, doi: 10.1016/j.cogsys.2018.05.004.

[9] Deng, J., Wang, H.-B., Wang, C.-M., Zhang, G.-W. (2017). A novel power market clearing model based on the equilibrium principle in microeconomics, Journal of Cleaner Production, Vol. 142, Part 2, 1021-1027, doi: 10.1016/ j.jclepro.2016.08.146. 
[10] Li, B., Lu, C., Cao, W., Qi, B., Li, D., Chen, S., Cui, G. (2017). Application of automatic demand response system based on blockchain technology, Chinese Journal of Electrical Engineering, Vol. 37, No. 13, 3691-3702, doi: 10.13334/j.0258-8013.pcsee.162462.

[11] Ai, X., Yang, M., Liu, Z., Li, X. (2017). Modelling and control safety of digital push-pull switched mode power supply, European Journal of Electrical Engineering, Vol. 19, No. 5-6, 341-355.

[12] Huckle, S., White, M. (2017). Fake news: A technological approach to proving the origins of content, using blockchains, Big Data, Vol. 5, No. 4, 356-371, doi: 10.1089/big.2017.0071.

[13] Hoy, M.B. (2017). An introduction to the blockchain and its implications for libraries and medicine, Medical Reference Services Quarterly, Vol. 36, No. 3, 273-279, doi: 10.1080/02763869.2017.1332261.

[14] Benchoufi, M., Porcher, R., Ravaud, P. (2017). Blockchain protocols in clinical trials: Transparency and traceability of consent, F1000 Research, Vol. 6, No. 66, doi: 10.12688/f1000research.10531.3.

[15] Suda, M., Tejblum, B., Francisco, A. (2017). Chain reactions: Legislative and regulatory initiatives related to blockchain in the United States, Computer Law Review International, Vol. 18, No. 4, 97-103, doi: 10.9785/cri2017-0402.

[16] Roth, U., Ngne Djoua, T. (2018). Message exchange on base of a blockchain-based layered architecture, ITInformation Technology, Vol. 60, No. 5-6, 253-261, doi: 10.1515/itit-2017-0037.

[17] Fu, Y., Zhu, J., Gao, S. (2018). CPS information security risk evaluation based on blockchain and big data, Tehnički Vjesnik - Technical Gazette, Vol. 25, No. 6, 1843-1850, doi: 10.17559/TV-20180621055030.

[18] Lu, Y. (2019). The blockchain: State-of-the-art and research challenges, Journal of Industrial Information Integration, Vol. 15, 80-90, doi: 10.1016/i.jii.2019.04.002.

[19] Jégou, D., Kim, D.-W., Baptiste, P., Lee, K.H. (2006). A contract net based intelligent agent system for solving the reactive hoist scheduling problem, Expert Systems with Applications, Vol. 30, No. 2, 156-167, doi: 10.1016/j.eswa. 2005.06.019.

[20] He, H.W., Yan, A., Chen, Z.H. (2018). Survey of smart contract technology and application based on blockchain, Journal of Computer Research and Development, Vol. 55, No. 11, 2452-2466, doi: 10.7544/issn1000-1239.2018. 20170658.

[21] Ping, J., Chen, S., Zhang, N., Yan, Z., Yao, L.Z. (2017). Decentralized transactive mechanism in distribution network based on smart contract, In: Proceedings of the Chinese Society of Electrical Engineering (CSEE), Vol. 37, No. 13, 3682-3690, doi: 10.13334/j.0258-8013.pcsee. 170374.

[22] Althaher, S., Mancarella, P., Mutale, J. (2015). Automated demand response from home energy management system under dynamic pricing and power and comfort constraints, IEEE Transactions on Smart Grid, Vol. 6, No. 4, 1874-1883, doi: 10.1109/TSG.2014.2388357.

[23] Yan, H., Li, B., Chen, S., Zhong, M., Li, D., Jiang, L., He, G. (2015). Future evolution of automated demand response system in smart grid for low-carbon economy, Journal of Modern Power Systems and Clean Energy, Vol. 3, No. 1, 72-81, doi: 10.1007/s40565-015-0103-5. 


\section{APEM}

ISSN 1854-6250

Spletna stran: apem-journal.org Izvirni znanstveni članek

\title{
Pametni pogodbeni trgovalni mehanizem z veriženjem blokov, za omrežje ponudbe in povpraševanja z energijo
}

\author{
Hu, W. ${ }^{a}$, Hu, Y.W. ${ }^{a}{ }^{*}$, Yao, W.H. ${ }^{a}$, Lu, W.Q. ${ }^{a}$, Li, H.H. ${ }^{a}$, Lv, Z.W. ${ }^{a}$ \\ ${ }^{a}$ School of Economics and Management, Shanghai University of Electric Power, Shanghai, P.R. China
}

\section{POVZETEK}

Da bi premagali visoke stroške, veliko tveganje in slabo učinkovitost tradicionalnega centraliziranega načina trgovanja $\mathrm{z}$ električno energijo, predlaga ta prispevek učinkovit trgovalni mehanizem za omrežje ponudbe in povpraševanja oskrbe z električno energijo (EPSDN), ki temelji na pametni pogodbi z veriženjem blokov, ob upoštevanju odprtja prodaje in liberalizaciji trga na Kitajskem. Natančneje, za določitev čakalne vrste in cene je bilo sprejeto pravilo spodbujanja realne kotacije (ERQ), s čimer se je uravnovesilo razmerje med ponudbo in povpraševanjem med vozlišči EPSDN. V transakcijo je bila uvedena pametna pogodba z veriženjem blokov, ki odpravlja centralizacijo in visoke stroške ter rešuje slabo preglednost in zaupanje $v$ tradicionalno transakcijo. Poleg tega je bila učinkovitost transakcij izboljšana z izgradnjo učinkovitega sistema trgovanja $\mathrm{z}$ električno energijo in varnega trgovinskega okolja. Na koncu prispevka je podana študija primera. Študija primera kaže, da sistem pametnega trgovanja s pogodbami EPSDN, ki temelji na veriženju blokov, lahko doseže željeno varnost in učinkovitost ter učinkovito reši težave tradicionalnega centraliziranega načina trgovanja. Ugotovitve raziskave podajajo teoretične in odločitvene podlage za manjše transakcije na trgu električne energije.

\section{PODATKI O ČLANKU}

Ključne besede:

Električna energija;

Omrežje ponudbe in povpraševan-

ja oskrbe z električno energijo

(EPSDN);

Veriženje blokov;

Pametna pogodba;

Pravilo spodbujanja realne kotacije

(ERQ);

Transakcija moči

*Kontaktna oseba: 99030411@qq.com (Hu, Y.W.)

Zgodovina članka:

Prejet 18. aprila 2019

Popravljen 7. septembra 2019

Sprejet 9. septembra 2019 